\title{
EDITORIAL
}

\section{Jinhao QIU \\ Introduction to the special section for ACMFMS 2010}

(C) Higher Education Press and Springer-Verlag Berlin Heidelberg 2011

Mechanics of functional materials and structures is an important area of research in mechanical engineering. The second Asian Conference on Mechanics of Functional Materials and Structures (ACMFMS 2010) was held in Nanjing from October 22 to 25, 2010 as a successor to the first ACMFMS, which was held in Matsue, Japan, in 2008. ACMFMS 2010 was sponsored by the Chinese Society of Theoretical and Applied Mechanics and the Jiangsu Society of Theoretical and Applied Mechanics and organized by Nanjing University of Aeronautics and Astronautics. It was organized to provide a forum for researchers and academics, particularly from East Asia and the neighboring regions, to present new findings, exchange ideas, and establish relationships, with an objective for future cooperation and subsequent promotion of research in theoretical and applied mechanics of solids. Young faculty members and researchers were especially encouraged to participate and use this opportunity to establish a network with others in this dynamic research field for skill improvement and experience enrichment.

The topics of the conference covered all aspects of research in functional materials and structures, including functional materials and fluids, bio-mimetic and bio-inspired materials, biomedical applications of smart materials and fluids, applications of smart materials in aerospace engineering, structural health monitoring technology, etc. 208 papers from 14 countries/regions were submitted to and presented in the conference. Among the presented papers, 20 were recommended to Frontier of Mechanical Engineering (FME). After peer review, 9 papers are accepted for publication in this special section.

I would like to acknowledge my appreciation to Prof. Cunfa Gao, the Chairman of ACMFMS 2010, Prof. Weiqiu Chen, the Co-Chairman, and Prof. Ji Wang for their kind help in paper screening for the special section.

Received July 10, 2011

Jinhao QIU (凶)

Guest Editor for the Special Section

State Key Laboratory of Strength and Vibration of Mechanical Structures, College of Aerospace Engineering, Nanjing University of Aeronautics and Astronautics, Nanjing 210016, China

E-mail: qiu@nuaa.edu.cn 